\title{
Giant Enhancement of Stimulated Brillouin Scattering in the Subwavelength Limit
}

\author{
Peter T. Rakich, ${ }^{1, *}$ Charles Reinke, ${ }^{1}$ Ryan Camacho, ${ }^{1}$ Paul Davids, ${ }^{1}$ and Zheng Wang ${ }^{2,3}$ \\ ${ }^{1}$ Sandia National Laboratories, P.O. Box 5800 Albuquerque, New Mexico 87185-1082, USA \\ ${ }^{2}$ Massachusetts Institute of Technology, 77 Massachusetts Avenue, Cambridge, Massachusetts 02139, USA \\ ${ }^{3}$ Department of Electrical and Computer Engineering, Microelectronics Research Center, The University of Texas at Austin, \\ Austin, Texas 78758 USA
}

(Received 22 August 2011; published 30 January 2012)

\begin{abstract}
Stimulated Brillouin scattering (SBS) is traditionally viewed as a process whose strength is dictated by intrinsic material nonlinearities with little dependence on waveguide geometry. We show that this paradigm breaks down at the nanoscale, as tremendous radiation pressures produce new forms of SBS nonlinearities. A coherent combination of radiation pressure and electrostrictive forces is seen to enhance both forward and backward SBS processes by orders of magnitude, creating new geometric degrees of freedom through which photon-phonon coupling becomes highly tailorable. At nanoscales, the backwardSBS gain is seen to be $10^{4}$ times greater than in conventional silica fibers with 100 times greater values than predicted by conventional SBS treatments. Furthermore, radically enhanced forward-SBS processes are $10^{5}$ times larger than any known waveguide system. In addition, when nanoscale silicon waveguides are cooled to low temperatures, a further 10-100 times increase in SBS gain is seen as phonon losses are reduced. As a result, a $100-\mu \mathrm{m}$ segment of the waveguide has equivalent nonlinearity to a kilometer of fiber. Couplings of this magnitude would enable efficient chip-scale stimulated Brillouin scattering in silicon waveguides for the first time. More generally, we develop a new full-vectorial theoretical formulation of stimulated Brillouin scattering that accurately incorporates the effects of boundary-induced nonlinearities and radiation pressure, both of which are seen to have tremendous impact on photonphonon coupling at subwavelength scales. This formalism, which treats both intermode and intramode coupling within periodic and translationally invariant waveguide systems, reveals a rich landscape of new stimulated Brillouin processes when applied to nanoscale systems.
\end{abstract}

DOI: 10.1103/PhysRevX.2.011008

\section{INTRODUCTION}

Stimulated Brillouin scattering (SBS) is a third-order nonlinear optical process by which optical photons are coupled to acoustic phonons. In conventional systems, SBS is mediated by electrostrictive or thermoelastic material response $[1,2]$. Through SBS, optically induced forces resulting from dynamic medium response drive the excitation of phonon modes that scatter light to new frequencies. Since its discovery [3], electrostrictively mediated stimulated Brillouin scattering has been extensively studied in bulk media and step-index optical fibers, enabling the realization of coherent phonon generation [3,4], slow light [5-8], efficient $\mathrm{GHz}$ phonon generation [3,9-12], and a host of new light sources [2,12-17] and signal-processing techniques $[2,5-8,18,19]$. In such systems, the strength of electrostrictive SBS is determined only by intrinsic material nonlinearities, arising from photoelastic material response, and is largely independent of the waveguide geometry $[1,2]$.

\footnotetext{
*rakich@alum.mit.edu.
}

Published by the American Physical Society under the terms of the Creative Commons Attribution 3.0 License. Further distribution of this work must maintain attribution to the author(s) and the published article's title, journal citation, and DOI.
Subject Areas: Nanophysics, Nonlinear Dynamics, Photonics

In translationally invariant waveguide systems, the conservation of momentum limits Brillouin scattering to one of two processes: (1) forward-SBS (FSBS), where both the incident and scattered waves are codirectionally coupled through transverse standing-wave phonons, and (2) backward-SBS (BSBS), where the incident and scattered waves are contradirectionally coupled through traveling-wave phonons. Forward-SBS processes are typically too weak to observe within conventional guided-wave systems $[2,20]$, and only recently has efficient FSBS coupling been observed through newly developed photonic crystal-fiber geometries, exhibiting improved lateral phonon confinement [13]. These systems have revealed cascaded Brillouin interactions with characteristics similar to Raman processes [13,21]. While this remarkable fiber geometry is the first to enable appreciable coupling via FSBS processes, the FSBS process (frequency 1-2 GHz) is significantly weaker than backward-SBS due to the superior confinement of longitudinal phonons. In such microstructured fibers, the transverse geometry of the fiber tailors the new phonon modes through which these SBS processes occur. However, at microscales, the optical forces which drive photon-phonon coupling predominantly arise from the nonlinearity of the electrostrictive material, fundamentally limiting the maximum strength of the SBS coupling. 
Through nanoscale modal confinement, strong interaction of light with the boundaries of a waveguide system can yield radically enhanced radiation pressures, produced independent of the intrinsic material nonlinearity [22-24]. The emergence of nano-optomechanics suggests that radiation pressure can be harnessed as a new way of creating strong photon-phonon coupling [22,23,25-34]. In particular, using simultaneous optical and phononic resonances, radiation-pressure-mediated photon-phonon couplings have enabled cryogenic optomechanical cooling [25,35], phonon lasers [36], and phononic signal processing [37-39].

Conventional treatments of SBS, which have proven remarkably accurate at microscales [1,2], do not account for radiation pressures or boundary-induced nonlinearities, which can have a very large effect at nanoscales. Hence, a unified treatment of photon-phonon coupling, capturing the contributions from both radiation pressure (a boundary-induced nonlinearity) and electrostriction (a bulk-material property), is necessary to elucidate the physics of SBS processes in nanoscale systems.

In this work, we develop a generalized multiscale treatment of photon-phonon coupling, valid at both microscales and nanoscales, which treats SBS coupling in both translationally invariant and periodic waveguide systems. This powerful full-vectorial treatment accurately captures phonon-mediated nonlinear coupling between any two optical modes through a Brillouin process. We apply this formalism to the study of traveling-wave SBS processes within nanoscale waveguides of the type seen in Fig. 1. Contrary to conventional wisdom, we show that, in the absence of material nonlinearities, enormous SBS nonlinearities are produced by large radiation pressures within nanoscale waveguides. Including the effects of material nonlinearity, radiation pressure and material-induced electrostrictive optical forces coherently combine to produce giant enhancement of both FSBS and BSBS processes, exceeding the SBS coupling of known systems by orders of magnitude. Additionally, the nontrivial interplay between electrostrictive and radiation-pressure-mediated photon-phonon coupling produces new geometric degrees of freedom, making the strength and frequency of photonphonon coupling highly tailorable.

Here, we find that the FSBS efficiency is greatly enhanced through nearly perfect lateral confinement of phonon modes, since the suspended waveguide geometry of Fig. 1 leaves no path for external phonon loss. In combination with highly confined phonon modes, a coherent combination of radiation pressure and electrostriction plays a critical role in the enhancement of FSBS processes. Employing the multiscale SBS theory presented here, we show that FSBS becomes the dominant Brillouin process at unique dimensions, yielding high-frequency (10-20 GHz) FSBS couplings which are $10^{5}$ times stronger than that of any other guided-wave system to date. As a consequence, such radically enhanced photon-phonon couplings could enable efficient stimulated Brillouin processes over remarkably short interaction lengths (approximately 100 microns). SBS couplings of this magnitude would enable efficient stimulated Brillouin scattering in silicon for the first time, leading to practical realization of slow light [5,6], dynamically tailorable effective susceptibilities [5,6], and nonreciprocal processes on optical chips [40].
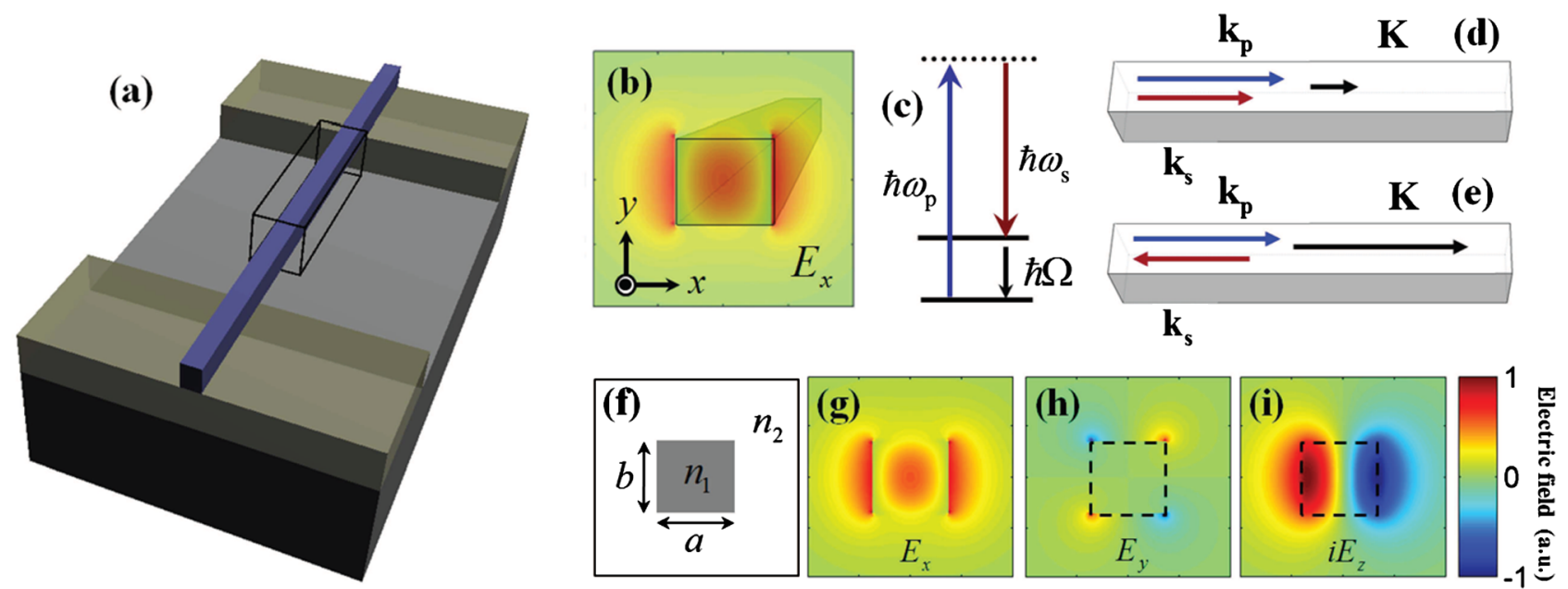

FIG. 1. (a) Sketch showing one experimental embodiment of a suspended silicon waveguide. The black wire-frame represents the portion of the waveguide examined here. (b) The cross-sectional sketch of the waveguide within the wire-framed region with the $E_{x}$ field of fundamental TE-like mode superposed. (c) The energy-level diagram of a Brillouin process. Here the optical pump and Stokes waves are shown in blue and red, respectively, while the phonon is shown in black. Phase-matching diagrams for forward (d) and backward (e) SBS. (f) The waveguide cross section (f) along with mode profiles for the (g) $E_{x}$, (h) $E_{y}$, and (i) $i E_{z}$ components of the fundamental TE-like mode for $\lambda=1550 \mathrm{nms}, a=300 \mathrm{~nm}$, and $b=280 \mathrm{~nm}$. 


\section{GENERALIZED TREATMENT OF SBS AT MICROSCALES AND NANOSCALES}

In typical Brillouin processes, a high-energy pump photon (of frequency $\omega_{\mathrm{p}}$ ) produces a red-shifted Stokes photon (of frequency $\omega_{\mathrm{s}}$ ) and an acoustic phonon (of frequency $\Omega$ ) via a third-order parametric process illustrated by the energy-level diagram in Fig. 1(c). Energy and momentum conservation require that $\omega_{\mathrm{p}}=\omega_{\mathrm{s}}+\Omega$, and $\mathbf{k}_{\mathrm{p}}=\mathbf{k}_{\mathrm{s}}+\mathbf{K}$, where $\mathbf{k}_{\mathbf{p}}, \mathbf{k}_{\mathbf{s}}$, and $\mathbf{K}$ are the pump, Stokes, and phonon wave vectors, respectively. Hence, phase-matching requires $\mathbf{k}_{\mathbf{p}}-\mathbf{k}_{\mathbf{s}}=\Delta \mathbf{k}=\mathbf{K}$. Within guided-wave systems, translational invariance limits the pump and Stokes-photon (and phonon) momenta to a single axis. Thus, the only possible scattering processes are FSBS, with colinear Stokes and pump waves, and BSBS where Stokes and pump waves are contradirectionally coupled (see Figs. 1(d) and 1(e)).

The magnitude of photon-phonon coupling through Brillouin processes can be completely modeled with rigorously coupled electromagnetic and elastic-wave models. Parametric coupling between photons and phonons is understood from interference between optical pump and Stokes waves, which produces a temporally modulated optical force distribution, $\mathbf{f}_{\boldsymbol{\Omega}}(\mathbf{r}, t)$, at beat frequency $\Omega$. The magnitude and distribution of this time-varying force distribution dictate the strength of photon-phonon coupling and the allowed $\Omega$ and $\mathbf{K}$ values. From these excited elastic modes, conventional SBS treatments determine the pump- and Stokes-wave coupling (or the Brillouin gain) from the nonlinear polarization currents (i.e., the time-derivative of the dielectric polarization) associated with elastic-wave motion in the waveguide core $[1,2]$. The effects of boundary-induced polarization currents and radiation pressures are generally neglected.

Such treatments of SBS are remarkably accurate at microscales. However, boundary-induced coupling cannot be neglected within nanoscale high-index-contrast waveguides, which exhibit high field concentrations about discontinuous dielectric boundaries [e.g., see Fig. 1(g)]. Furthermore, application of conventional polarizationcurrent analyses becomes challenging at nanoscales, as polarization currents are ill defined within complex geometries where both electric field and dielectric profiles are discontinuous [41]. Moreover, since radiation-pressureinduced nonlinearities are not treated, these models fail to capture essential mechanisms of photon-phonon coupling at nanoscales.

To address these challenges, we develop a formalism to treat photon-phonon coupling accurately at any length scale. By employing phononic-domain calculations, this new multiscale theory of photon-phonon coupling avoids the difficulties of formulating nonlinear polarization currents within complex nano-optomechanical systems. We instead compute the elastic-wave power generated by optical forces. Invoking particle and energy conservation, which are well established within a quantum framework
[42], we then show that an exact formulation of SBS gain can be made directly from the optically generated elasticwave power. In addition to yielding greatly simplified treatment of SBS in complex nano-optomechanical structures, this model enables the treatment of all optical force contributions in a single model.

Through guided-wave Brillouin processes, the growth of the optical Stokes wave through parametric conversion between pump and Stokes waves is quite generally described by the canonical relation $[1,2]$

$$
d P_{\mathrm{s}} / d z=G_{B} \cdot P_{\mathrm{p}} P_{\mathrm{s}}-\alpha_{\mathrm{s}} P_{\mathrm{s}} .
$$

Here, $P_{\mathrm{p}}$ and $P_{\mathrm{s}}$ are the guided powers of the pump and Stokes waves respectively, $G_{\mathrm{B}}$ is the stimulated Brillouin scattering modal gain coefficient, and the Stokes-wave propagation loss-factor is $\alpha_{\mathrm{s}}$, with propagation along the $z$-axis. In this treatment, we assume that optical modes carrying powers $P_{\mathrm{p}}$ and $P_{\mathrm{s}}$, with associated optical frequencies $\omega_{\mathrm{p}}$ and $\omega_{\mathrm{s}}$, are coupled by optically generated acoustic phonons of frequency $\Omega$ and power $P_{\Omega}$.

Note that intermode coupling, or coupling between dissimilar optical modes, can occur through Brillouin interactions. For instance, in Ref. [43], Kang et al. demonstrate strong coupling between cross-polarized modes. To treat these and other cases of intermode coupling, the pump wave $\left(P_{\mathrm{p}}\right)$ and Stokes wave $\left(P_{\mathrm{s}}\right)$ may be assumed to occupy similar or dissimilar guided optical modes. The following treatment applies identically for both cases.

We begin by recasting Eq. (1) in terms of particle fluxes. In the classical limit, $P_{\mathrm{p}}, P_{\mathrm{s}}$, and $P_{\Omega}$ coincide with particle fluxes $\Phi_{\mathrm{p}}, \Phi_{\mathrm{s}}$, and $\Phi_{\Omega}$, respectively. Dividing both sides of Eq. (1) by the Stokes-photon energy, $\hbar \omega_{\mathrm{s}}$, we have

$$
d \Phi_{\mathrm{s}} / d z=\gamma_{\mathrm{s}}-\alpha_{\mathrm{s}} \Phi_{\mathrm{s}} .
$$

Above, $\gamma_{\mathrm{s}}=G_{\mathrm{B}} P_{\mathrm{p}} P_{\mathrm{s}} / \hbar \omega_{\mathrm{s}}$, where $\gamma_{\mathrm{s}}$ is defined as the Stokes-photon-generation rate per unit length, and $\hbar$ is Planck's constant. From established quantum-mechanical treatments of Brillouin scattering [42], particle conservation requires $\gamma_{\mathrm{s}}=\gamma_{\Omega}=-\gamma_{\mathrm{p}}$, where $\gamma_{\mathrm{p}}$ and $\gamma_{\Omega}$ are the pump photon and phonon generation rates per unit length. This relation is also seen from the quantum-mechanical energy-level diagram of Fig. 1(c).

Relating this particle-generation rate to classical fieldbased quantities, we see that $\gamma_{\Omega}=\left(\delta P_{\Omega} / \delta z\right)(1 / \hbar \Omega)$, where $\delta P_{\Omega}$ is the elastic-wave power generated in a short segment, $\delta z$, of the waveguide. Since the instantaneous elastic-wave power density generated by optical forces within the waveguide is given by $\mathbf{f}_{\boldsymbol{\Omega}} \cdot \dot{\mathbf{u}}_{\boldsymbol{\Omega}}$, the timeaveraged particle generation rate, $\gamma_{\Omega}$, is readily computed as

$$
\gamma_{\Omega}=\frac{1}{\delta z} \frac{1}{\hbar \Omega} \int_{\delta V}\left\langle\mathbf{f}_{\mathbf{\Omega}}(\mathbf{r}, t) \cdot \dot{\mathbf{u}}_{\mathbf{\Omega}}(\mathbf{r}, t)\right\rangle d V .
$$

Here, $\dot{\mathbf{u}}_{\Omega}(\mathbf{r}, t)$ is the displacement velocity distribution of the elastic body in response to $\mathbf{f}_{\boldsymbol{\Omega}}(\mathbf{r}, t),\langle\ldots\rangle$ denotes time 
averaging over an acoustic period, and integration is taken over $\delta V$, the volume of the waveguide segment. Hence, in the limit of small $\delta z, G_{\mathrm{B}}$ is given by

$$
G_{\mathrm{B}}(\Omega)=\frac{1}{\delta z} \frac{\omega_{\mathrm{s}}}{\Omega} \cdot \frac{1}{P_{\mathrm{p}} \cdot P_{\mathrm{s}}} \int_{\delta V}\left\langle\mathbf{f}_{\mathbf{\Omega}}(\mathbf{r}, t) \cdot \dot{\mathbf{u}}_{\mathbf{\Omega}}(\mathbf{r}, t)\right\rangle d V .
$$

Note that, for systems with continuous translational symmetry, Eq. (4) yields an exact formulation of SBS gain in the limit as $\delta z \rightarrow 0$. Furthermore, it bears mentioning that the derivation of Eq. (4) does not place any limitations on the longitudinal or transverse structure of the system. Thus, Eq. (4) can also be used to determine the effective SBS gain of a periodic system when integration, $\delta V$, is taken over a single unit cell.

It should be noted that Eq. (4) can be used to treat photon-phonon coupling accurately at any length scale, provided that a valid model of the optical forces and elastic-wave motion can be formulated. Furthermore, use of particle conservation in deriving Eq. (4) is entirely general, provided that the elastic equation of motion is in the linear regime. Both optical and mechanical losses are faithfully treated by this model, since particle conservation is used only to capture the particle-generation process. Throughout, we assume that $\mathbf{f}_{\boldsymbol{\Omega}}(\mathbf{r}, t)$ is a generalized optical-force density, including all or any time-varying optical forces. As a point of reference, exact SBS solutions are readily computed in numerous cases from Eq. (4), revealing perfect agreement with conventional SBS treatments. For instance, we show in Appendix A that the conventional BSBS gain relation for optical fibers can be exactly retrieved from Eq. (4).

Limiting our attention to the special case of a translationally invariant waveguide, it is useful to express the force and velocity distributions in complex notation as

$$
\begin{gathered}
\mathbf{f}_{\boldsymbol{\Omega}}(\mathbf{r}, t)=\operatorname{Re}\left[\tilde{\mathbf{f}}_{\boldsymbol{\Omega}}(\mathbf{x}, \mathbf{y}) \cdot e^{i(K z-\Omega t)}\right], \\
\dot{\mathbf{u}}_{\boldsymbol{\Omega}}(\mathbf{r}, t)=\operatorname{Re}\left[\dot{\tilde{\mathbf{u}}}_{\boldsymbol{\Omega}}(\mathbf{x}, \mathbf{y}) \cdot e^{i(K z-\Omega t)}\right] .
\end{gathered}
$$

Defining power-normalized force and velocity distributions, $\tilde{\mathbf{f}}_{\mathbf{n}}(\mathbf{x}, \mathbf{y})$ and $\dot{\tilde{\mathbf{u}}}_{\mathbf{n}}(\mathbf{x}, \mathbf{y})$ such that $\tilde{\mathbf{f}}_{\mathbf{\Omega}}(\mathbf{x}, \mathbf{y})=$ $\tilde{\mathbf{f}}_{\mathbf{n}}(\mathbf{x}, \mathbf{y}) 2 \sqrt{P_{\mathrm{s}} P_{\mathrm{p}}}$, and $\dot{\tilde{\mathbf{u}}}_{\mathbf{\Omega}}(\mathbf{x}, \mathbf{y})=\dot{\tilde{\mathbf{u}}}_{\mathbf{n}}(\mathbf{x}, \mathbf{y}) 2 \sqrt{P_{\mathrm{s}} P_{\mathrm{p}}}$, we can simplify Eq. (4) to

$$
G_{B}(\Omega)=2 \cdot\left(\frac{\omega_{s}}{\Omega}\right) \int_{\mathrm{wg}} \operatorname{Re}\left[\tilde{\mathbf{f}}_{\mathbf{n}}(\mathbf{x}, \mathbf{y}) \cdot \dot{\tilde{\mathbf{u}}}_{\mathbf{n}}^{*}(\mathbf{x}, \mathbf{y})\right] d A .
$$

Here, integration is taken over the waveguide cross section. Since the vector-force distribution, $\tilde{\mathbf{f}}_{\mathbf{n}}(\mathbf{x}, \mathbf{y})$, can be readily computed from the interference between any two pumpand Stokes-wave field distributions, this full-vectorial formulation of SBS allows for exact treatment of intramode and intermode SBS coupling. For further details regarding the general form of $\tilde{\mathbf{f}}_{\mathbf{n}}(\mathbf{x}, \mathbf{y})$, see Appendix B. Equation (7) applies to translationally invariant waveguides of arbitrary cross section. Furthermore, since the force density is quite generally defined, both Eqs. (4) and (7) can be applied to treat the effects of radiation pressure, electrostriction, thermoelastic material response, or any other power-dependent force.

For generality, we have not limited our discussion to a particular form of the elastic-wave equation, as the intrinsic losses and tensor properties of various elastic media can result in one of many equations of motion [44]. For instance, the commonly used Navier-Stokes equation is valid in isotropic elastic media, but alternate wave equations must be used in anisotropic media [44].

Finally, since elastic-wave damping plays an important role in determining the maximum value of the SBS gain, it bears mentioning that, on resonance, the peak value of the elastic-displacement velocity is proportional to the acoustic quality factor, $Q$, and the magnitude of the optical force. Hence, for a linear elastic system with resonances which are directly coupled by the optical forces, one can show that $G_{\mathrm{B}}$ scales as $G_{\mathrm{B}} \propto Q\left|\mathbf{f}_{\mathbf{n}}\right|^{2}$. Thus, it is compelling to explore the scaling and enhancement of SBS within high refractive-index waveguides of subwavelength dimension, where optical forces are greatly enhanced $[23,31]$.

\section{TREATMENT OF SBS IN A NANOSCALE SILICON WAVEGUIDE}

Next, we examine SBS within a nanoscale waveguide by applying the full-vectorial multiscale SBS formalism of Sec. II. Throughout this study, we consider SBS within the silicon waveguide geometry of Fig. 1, corresponding to a core (cladding) refractive index of $n_{1}=3.5\left(n_{2}=1\right)$ and material photoelastic constants $\left[p_{11}, p_{12}, p_{44}\right]=$ $[+0.09,-0.017,-0.054]$ consistent with those of bulk silicon [45]. Here, $p_{i j}$ are the photoelastic-tensor components $\left(p_{i j k l}\right)$ represented in contracted notation [46], and the [100] symmetry direction of this crystalline silicon waveguide coincides with the $x$ axis.

Characteristic field profiles of the transverse-electriclike (TE-like) mode under consideration are seen in Fig. 1. We will limit our attention to intramode SBS processes where both pump and Stokes waves coincide with this fundamental TE-like waveguide mode. However, in general, the treatment in Sec. II can be used to examine intermode SBS coupling between any two waveguide modes that have arbitrary spatial distribution and polarization.

For nanoscale silicon waveguides of the type seen in Fig. 1, it has been shown that both radiation pressure and electrostrictive forces grow by orders of magnitude as the waveguide dimensions approach the nanoscale $[23,24]$. Because of the high waveguide dispersion, which is closely linked to the fractional energy density carried by the 
longitudinal $\left(E_{z}\right)$ electric field [47], the power-normalized values of radiation pressure grow to values which are $10^{2}-10^{4}$ times larger than those within optical fibers [23]. As a result, forces from radiation pressure can exceed those of electrostriction at nanoscales [24].

The distributions of electrostrictive- and radiationpressure-induced forces are distinct within nanoscale waveguides. Radiation-pressure-induced optical forces result from the scattering of light at boundaries, producing forces exactly localized to the discontinuous dielectric boundary of the step-index waveguide $[23,24,48]$. Conversely, electrostrictive forces result from the dynamic mechanical response of media to light, acting through the strain degrees of freedom of a medium. The magnitude and orientation of electrostrictive forces are determined by the bulk photoelastic-material tensors, producing complex spatially varying body-force distributions within the waveguide volume [24]. Both forces depend sharply on nanoscale waveguide geometry and yield vanishing longitudinal forces under single-mode excitation of a translationally invariant waveguide [23,24].

In treating photon-phonon coupling through SBS, we examine the temporally modulated component of the optical force which drives the SBS process. This dynamic analysis of force must not be confused with the static analyses of force treated in Refs. [23,24]. In contrast to the single-mode cases of previous studies, the interference between pump and Stokes waves produces a time-varying transverse force density that diverges significantly from the single-mode case. Mode-interference gives rise to additional longitudinal force densities which are not present under single-mode excitation. For colinear pump and Stokes waves (FSBS), the time-varying part of the transverse force density reduces to an approximate form similar to that described in [24]. However, in the contradirectional case (BSBS), wave interference produces a temporally modulated force density that has a markedly different spatial distribution for both radiation pressure and electrostriction. Most notably, a large longitudinal electrostrictive force is produced in the contradirectional case, which is absent in the colinear case. For further details, see the general form of the time-harmonic force density, $\tilde{\mathbf{f}}_{\mathbf{\Omega}}(\mathbf{x}, \mathbf{y})$, given in Appendix B.

To evaluate SBS within this waveguide, we derive the general analytical form of the complex time-harmonic optical-force distribution, $\tilde{\mathbf{f}}_{\boldsymbol{\Omega}}(\mathbf{x}, \mathbf{y})$, and use it to compute the time-varying optical-force density in conjunction with an electromagnetic finite-element-method (FEM) model. The detailed procedure is outlined in Appendix B. These force densities serve as the time-harmonic forcing function
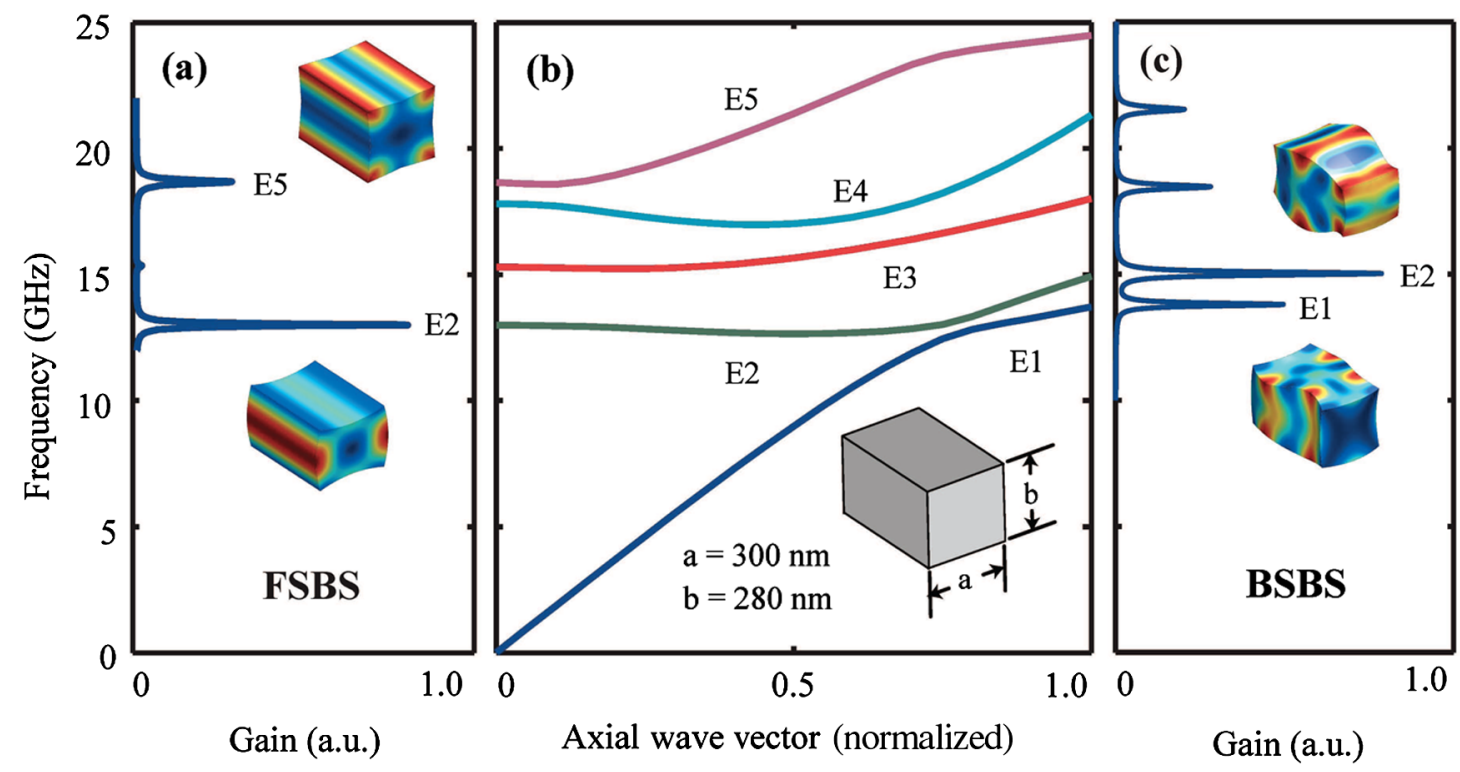

FIG. 2. Computed SBS gain shown in arbitrary units (a.u.) for (a) FSBS and (c) BSBS processes, for comparison with the computed phonon dispersion of the lowest-order symmetric phonon modes (labeled E1-E5) seen in (b). The FSBS and BSBS resonances match well with computed dispersion of (b). In (b), the wave vector is normalized by $K=2 k_{\mathrm{p}}$ for wavelength $\lambda=1550 \mathrm{nms}$. Colored insets of (a) show the displacement amplitudes and shape deformation of the dominant E2 and E5 phonons about $K=0$ excited through FSBS, while those of (c) show the E1 and E2 modes at $K=2 k_{\mathrm{p}}$ in the BSBS case. High (low) displacement regions are shaded red (blue). Calculations assume silicon waveguide dimensions of $a=300 \mathrm{~nm}$ and $b=280 \mathrm{~nm}$ and wavelength $\lambda=1550 \mathrm{nms}$. Note, elastic modes which do not contribute to SBS have been omitted from the dispersion plot in (b). For clarity, the computed elastic modes seen in (b) were restricted to those with reflection symmetries about the $x-z$ and $y-z$ mirror planes centered on the waveguide core. However, the frequency dependent elastic-wave models used to compute SBS gain in (a) and (c) do not limit the number of modes by use of symmetry. Hence, SBS gain shown in (a) and (c) includes the contribution to photon-phonon coupling arising from all acoustic modes in the system. 
within an elastic-wave FEM model of the waveguide, yielding the SBS gain, $G_{\mathrm{B}}(\Omega)$, vs phonon frequency, by use of Eq. (7). Throughout, we use Young's modulus, $E=170 \times 10^{9} \mathrm{~Pa}$, Poisson's ratio, $\nu=0.28$, and density, $\rho=2329 \mathrm{~kg} / \mathrm{m}^{2}$ to model silicon. For simplicity, a frequency-independent phononic-material loss, $Q$, of 1000 is assumed, as this $Q$ is below the damping limit of silicon for the frequency range of interest [49]. For details regarding solution of the elastic-wave equation by FEM models, and the implementation of elastic-wave damping, see Ref. [50].

Since the TE-like mode results in symmetric force distributions from both electrostriction and radiation pressure, one finds that only symmetric elastic waves can be excited through either the FSBS or the BSBS processes considered here. The elastic-wave dispersion of the first five symmetric elastic modes (labeled E1-E5) are plotted in Fig. 2(b) for comparison with the computed FSBS and BSBS gain spectra of Figs. 2(a) and 2(c). In the next sections, we present detailed analyses of FSBS and BSBS, and the novel mechanisms which are unique to nanoscale photon-photon coupling.

\section{NANOSCALE FORWARD SBS}

In conventional guided-wave systems, forward SBS is typically orders of magnitude weaker than backward SBS [20]. Phase matching requires the phonon wave vector $|\mathbf{K}| \cong \Omega / v_{g}$, where $v_{g}$ is the group velocity of the guided optical mode. Consequently, the longitudinal phonon wave vector, $|\mathbf{K}|$, is nearly zero, resulting in the excitation of guided elastic waves with vanishing group velocity (or resonances). These waves tend to be weakly confined low-frequency modes in conventional step-index fiber systems, yielding weak photon-phonon coupling [20]. In contrast to microscale fiber-optic systems, nearly perfect lateral confinement of the $|\mathbf{K}| \cong 0$ phonon modes is achieved in this silicon-waveguide geometry, contributing
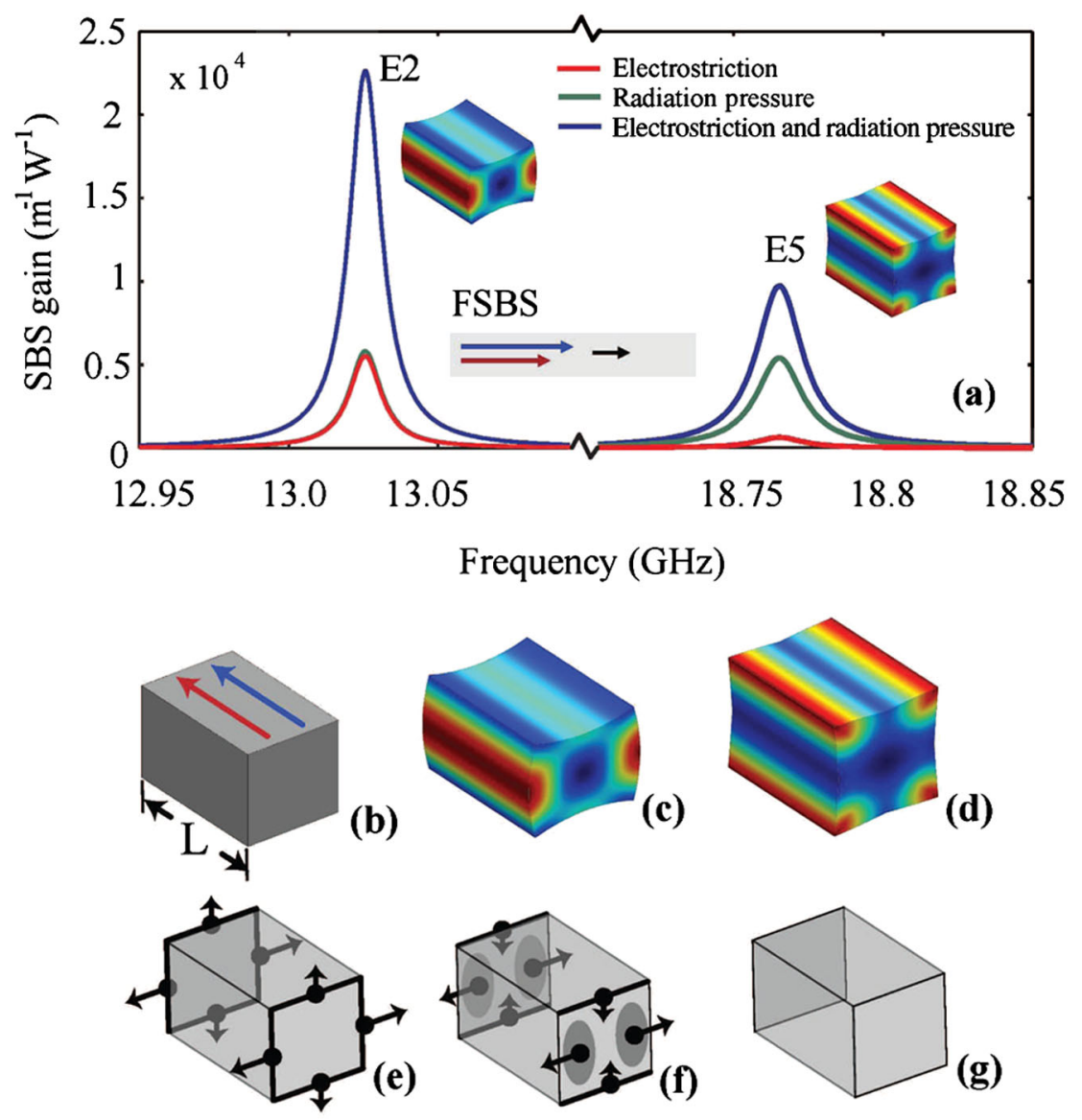

FIG. 3. Detailed analysis of FSBS: (a) The computed FSBS gain vs the phonon frequency near the E2 and E5 resonances centered at 13.0 and $18.8 \mathrm{GHz}$. The individual contributions of electrostriction (red) and radiation pressure (green) to the SBS gain are shown along with their combined effect (blue). Simulations assume a phononic loss $Q$ of 1000 and optical wavelength $\lambda=1.55 \mu \mathrm{ms}$. (b) A schematic of a waveguide segment $(L=300 \mathrm{~nm})$. (c) and (d) are the characteristic displacements of the E2 and E5 elastic modes. High-displacement regions are shown in red, and low-displacement regions are shown in blue. Schematics showing the amplitudes and directions of the dominant components of $\tilde{\mathbf{f}}_{\mathbf{\Omega}}(\mathbf{x}, \mathbf{y})$ are seen in (e), (f), and (g). Radiation pressure contributes only to the transverse force components (e), while electrostriction contributes to both the transverse components (f) and the longitudinal components (g). Note that the longitudinal forces are vanishingly small for FSBS. 
to far stronger photon-phonon coupling in the FSBS case. Comparison of the computed FSBS gain of Fig. 2(a) with the dispersion plot of Fig. 2(b) reveals that shear and compressive standing-wave resonances associated with E2 and E5 are predominantly excited via FSBS. The characteristic modal displacements of the dominant modes are seen as insets in Fig. 2(a).

Closer examination of the photon-phonon coupling within this system reveals that both radiation pressure and electrostriction play an important role in the SBS process at nanoscales. A magnified view of the computed FSBS gain is seen in Fig. 3(a) for resonances corresponding to the E2 and E5 elastic modes at 13.0 and $18.8 \mathrm{GHz}$, respectively. The graph shows individual contributions of electrostriction (red curve) and radiation pressure (green curve) to the SBS gain, along with their combined effect (blue curve) due to the coherent combination of radiation pressure and electrostriction.

The contributions from electrostriction and radiation pressure are mode specific. While electrostriction and radiation pressure produce nearly identical coupling to the E2 shear-mode, radiation pressure is seen to be the dominant source of coupling to the E5 mode. Notably, the coherent addition of electrostriction and radiation pressure dramatically enhances the SBS gain, yielding a total gain of $2.3 \times 10^{4} \mathrm{~m}^{-1} \mathrm{~W}^{-1}$ at $13.0 \mathrm{GHz}$, more than $10^{4}$ times larger than that of recently studied photonic crystal-fiber systems [13]. As will be discussed in Sec. VII, the loss characteristics of the E2 shear mode are likely to be far superior to the uniform loss-estimate applied here, yielding SBS gains which are 3 times larger than the simulations of Fig. 3 indicate.

The coherent interaction between electrostriction and radiation pressure can be understood from the internally generated optical forces. In the case of colinear pump and Stokes waves, the longitudinal electrostrictive forces are found to be vanishingly small. Thus, transverse forces of radiation pressure and electrostriction almost exclusively dictate SBS coupling in the FSBS case.

The dominant components of optical force produced by electrostriction and radiation pressure [generated within the waveguide segment of Fig. 3(b)] are sketched in Figs. 3(e)-3(g) for comparison with the elastic mode displacements of Figs. 3(c) and 3(d). Radiation pressure induces outward forces which are localized to the boundaries of the waveguide, while electrostriction produces a combination of inward and outward forces within the volume of the waveguide. This unusual electrostrictive-force distribution sharply contrasts with silica fibers, which yield only a single sign of force in the transverse dimension. The existence of inward and outward forces results from the differing signs of the $p_{11}$ and $p_{12}$ in silicon. Notably, the distribution of these forces coherently combines to yield a very efficient excitation of the shear elastic-wave (or wine-glass) resonance associated with E2, which is known to exhibit remarkably low damping in phononic systems [49].

Since the dominant components of force for both electrostriction and radiation pressure coherently combine in the lateral (or $x$ ) direction, it is apparent that E2 is most efficiently excited. On the other hand, the outward forces of radiation pressure are almost exclusively responsible for efficient excitation of the compressive E5 elastic mode. Hence, the nontrivial interplay between electrostriction and radiation pressure significantly complicates the photon-phonon coupling, warranting further study in nanoscale systems.

Since the distributions of optical force from electrostriction and radiation pressure become highly dependent on geometry at nanoscales [24], it should be noted that these new geometric degrees of freedom make it possible to tailor the SBS process to an unprecedented degree. Optomechanical phonon frequency, mode shape, and coupling strength are all seen to vary sharply with the waveguide geometry.

\section{NANOSCALE BACKWARD SBS}

In the BSBS case, phase matching requires that $\Delta \mathbf{k}=$ $\mathbf{K} \cong 2 \cdot \mathbf{k}_{\mathbf{p}}$. Comparison of the computed FSBS gain of Fig. 2(c) with the dispersion plot of Fig. 2(b) near $\mathbf{K}=2 \cdot \mathbf{k}_{\mathbf{p}}$ reveals that traveling elastic modes, E1 and $\mathrm{E} 2$, with both longitudinal and transverse displacements are dominant through BSBS [see insets in Fig. 2(c)].

A magnified view of the computed BSBS gain spectrum is seen in Fig. 4(a) near the E1 and E2 elastic-wave resonances at 13.8 and $15.0 \mathrm{GHz}$, respectively. Again, the individual contributions of electrostriction (red curves) and radiation pressure (green curves) to the SBS gain are shown, along with their combined effect (blue curves) due to the coherent combination of radiation pressure and electrostriction. Electrostriction and radiation pressure constructively interfere, yielding a dominant coupling to the $15.02 \mathrm{GHz}$ resonance, with an SBS gain of $1.3 \times 10^{4} \mathrm{~m}^{-1} \mathrm{~W}^{-1}$. This result is more than $10^{4}$ times larger than in typical optical fibers.

The dominant components of electrostrictive and radiation-pressure-induced forces, which mediate BSBS, are sketched in Figs. 4(e)-4(g) for comparison with the elastic-mode displacements of Figs. 4(c) and 4(d) within the waveguide segment seen in Fig. 4(b). In the BSBS case, a large $\Delta k$ results in rapid spatial modulation of all optical forces in the longitudinal direction. It should also be noted that modal interference between counterpropagating pump and Stokes waves generates new longitudinal electrostrictive-force components of comparable magnitude to the transverse forces generated by both radiation pressure and electrostriction. Alternatively, from the fundamental functional form of radiation pressure [48], one can show that, in contrast to electrostriction, radiation pressure 

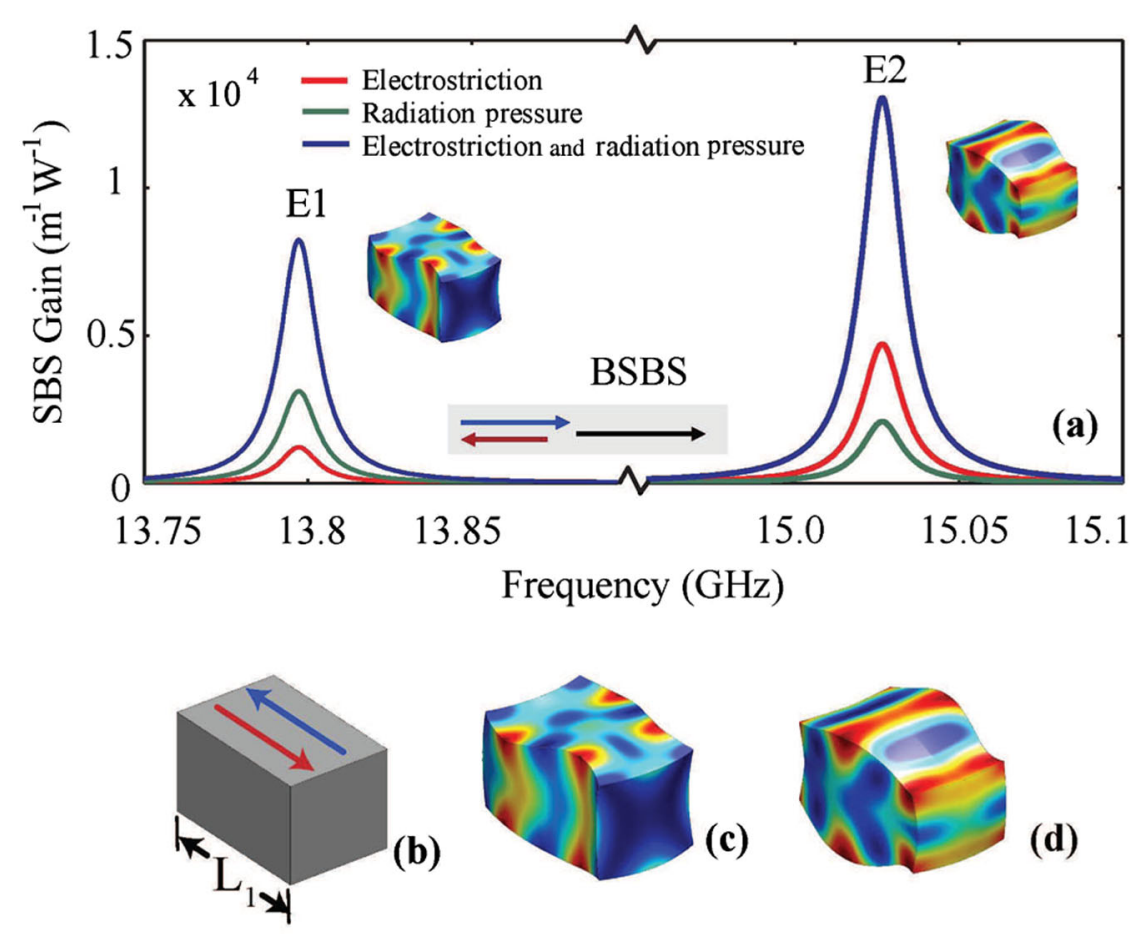

(d)
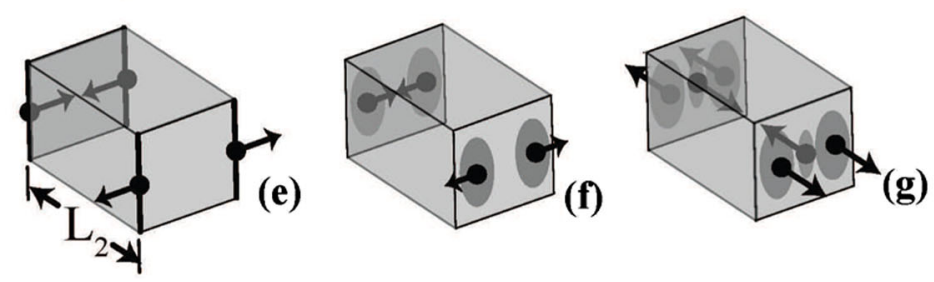

FIG. 4. Detailed analysis of BSBS: (a) The computed BSBS gain vs the phonon frequency near the E1 and E2 resonances centered at 13.8 and 15.0 GHz. The individual contributions of electrostriction (red) and radiation pressure (green) to the SBS gain are shown along with their combined effect (blue). Simulations assume a phononic loss $Q$ of 1000 and optical wavelength $\lambda=1.55 \mu$ m. (b) A schematic of a waveguide segment $\left(L_{1}=300 \mathrm{~nm}\right)$, and characteristic mode displacements (c) and (d) of the E1 and E2 elastic modes. High-displacement regions are shown in red, and low-displacement in blue. Schematics (e), (f), and (g) show the amplitudes and directions of the dominant components of $\tilde{\mathbf{f}}_{\boldsymbol{\Omega}}(\mathbf{x}, \mathbf{y})$ within a waveguide segment of length $L_{2}=(\pi / \Delta k) \cong 150 \mathrm{~nm}$. Radiation pressure contributes only to the transverse force components (e), while electrostriction contributes to both the transverse components (f) and the longitudinal components (g).

does not produce longitudinal forces within the axially uniform waveguide examined here.

Interference between the two modes also significantly alters the transverse character of both radiation pressure and electrostriction. As seen in Figs. 4(e) and 4(f), $y$-directed forces are greatly diminished in comparison with the FSBS case. In the BSBS case, one finds that the transverse forces from radiation pressure are dominant in exciting the transverse elastic wave (E1) seen in Fig. 4(c), and longitudinal electrostrictive forces primarily drive the longitudinal (E2) elastic resonance of Fig. 4(d).

Based on the value of $p_{12}$ and the refractive index of silicon, commonly used scalar BSBS models treat SBS coupling as a bulk material property of the system, underpredicting the SBS gain by a factor of 100 . For further details, see Appendix A. As we will discuss in Sec. VI, these results indicate that, at nanoscales, SBS gain can no longer be interpreted as a bulk-material property, as is commonly done at microscales.

\section{SCALING OF SBS WITH DIMENSION}

Since the FSBS and BSBS processes discussed in Secs. IV and V show little resemblance to those of optical fibers, it is apparent that SBS becomes highly geometrydependent at subwavelength scales. To explore the scale dependence of SBS further, we examine the variation in SBS gain with the transverse dimension of the waveguide. Figures 5(a) and 5(b) show the contributions of electrostriction and radiation pressure to the total BSBS and FSBS gains, respectively, as the waveguide dimensions vary between $0.2-10 \mu \mathrm{ms}$. Each simulation point represents the maximum value of the computed SBS gain among all phonon modes between 0-50 GHz. The radiation-pressure contribution to SBS (green curves) was computed by 

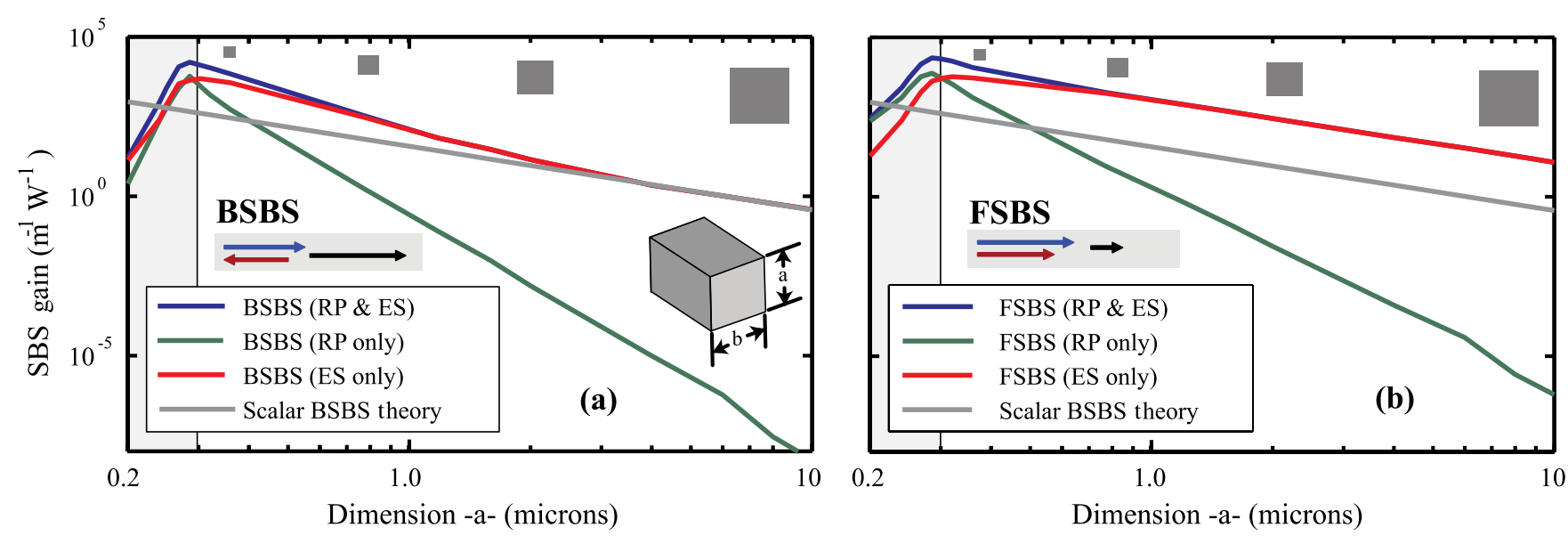

FIG. 5. Computed SBS contributions from electrostriction (ES) and radiation pressure (RP) as the waveguide dimension, $a$, is varied from 0.2-10 microns (for $b=0.93 a$ ). (a) BSBS gain resulting from ES (red), RP (green), and the coherent combination of both ES and RP (blue). (b) An identical set of curves for FSBS. In comparison, the conventional microscale SBS theory (gray curves) underestimates the BSBS gain by up to 2 orders of magnitude for length scales between $250 \mathrm{~nm}$ and a few microns. For further details on the microscale SBS trend (gray curves), see Appendix A. The dimensions where radiation pressure is dominant are shaded gray.

setting the photoelastic constants to zero, and the electrostrictive contribution (red curves) was computed by neglecting the optical forces associated with radiation pressure (i.e., the force terms derived from the Maxwell stress tensor).

To highlight the new physics captured by the multiscale SBS model of Sec. II, it is useful to compare this model with existing SBS treatments over a range of length scales. Here, we do not seek to compare our full-vectorial multiscale model with the numerous accepted treatments which precede ours. Detailed comparison warrants significant further discussion, which is beyond the scope of this paper. We instead choose to explore the manner in which subwavelength modal confinement radically alters SBS by comparison with SBS treatments that are familiar to most in the nonlinear-optics community $[1,2]$.

The gray lines of Figs. 5(a) and 5(b) show the SBS gain predicted by the widely used scalar-SBS treatment, which is known to be very accurate in microscale systems. This formulation assumes acousto-optic coupling between only the transverse-electric fields and longitudinal elastic displacement (through tensor element $p_{12}$ ), and uses mode overlaps to approximate the SBS gain. For further details, see Appendix A. While the assumptions of this model break down at smaller length scales, this model allows us to establish correspondence with accepted SBS models, and it highlights the basic dimensional dependence of electrostrictively mediated Brillouin processes.

From Fig. 5(a), we see perfect agreement between the models in the BSBS case for dimensions greater than $4 \mu \mathrm{m}$, demonstrating convergence with well-established SBS treatments at microscales. However, for smaller di- mensions, this simplified scalar SBS theory is seen to underpredict the total BSBS gain by a factor of 100 , revealing the importance of more complete $a b$ initio modeling in this regime. It is instructive to note that the peak BSBS gain predicted by electrostrictive interactions $(3.2 \times$ $\left.10^{3} \mathrm{~m}^{-1} \mathrm{~W}^{-1}\right)$ is approximately 25 times larger than that predicted by the scalar SBS treatment (see Sec. VI). This disparity becomes 4 times larger when we include the effects of radiation pressure, yielding a 100-times underprediction of SBS gain at small dimensions.

Divergence from the microscale model is expected as a result of radiation pressure, since radiation pressure is not included in previous SBS treatments. However, the discrepancy in SBS gain arising from electrostrictive interactions is more subtle in origin. To understand the disparity in the predicted electrostrictive coupling, we note that a large fraction of the mode energy is carried by the $E_{z}$ field at nanoscales, causing the electrostrictive forces to depend strongly on $p_{11}$ and $p_{44}$ photoelastic constants, in addition to the $p_{12}$ coefficient (which governs BSBS at microscales). Since the $p_{11}$ is more than 5 times larger than $p_{12}$ in silicon, coupling of electrostrictive forces through $p_{11}$ significantly increases the BSBS gain. A further increase in electrostrictive coupling results from dispersioninduced enhancement of the electric-energy density at small dimensions.

In examining the FSBS calculations in Fig. 5(b), it should be noted that the electrostrictive component of FSBS gain (red curve) is orders of magnitude larger than that of the microscale BSBS trend (gray line) for all dimensions. This is because the transverse electrostrictive forces that mediate FSBS predominantly couple through 
the $p_{11}$ photoelastic constant, which produces much stronger coupling than the $p_{12}$ constant that is used to model BSBS in widely employed scalar SBS theories.

Inspecting the effects of radiation pressure on SBS, we see that negligible contributions to the total BSBS and FSBS gains are produced by radiation pressure at large dimensions. However, the radiation-pressure component to SBS increases very sharply at smaller dimensions, overtaking that of electrostriction where the waveguide dispersion reaches a maximum $(a \cong 300 \mathrm{~nm})$ in both cases. The electrostrictive and radiation-pressure contributions to the FSBS and BSBS gains are seen to roll off for $a<250 \mathrm{~nm}$, as modal confinement becomes very weak.

The scale dependence of electrostrictive- and radiationpressure-induced SBS couplings are seen to be quite distinct in Figs. 5(a) and 5(b). Since the microscale model approximates the SBS coupling as an intrinsic property of the waveguide material, the SBS gain predicted by the microscale-SBS trend scales as $A_{\mathrm{wg}}^{-1}$. Here, $A_{\mathrm{wg}}$ is the cross-sectional area of the waveguide given by $A_{\mathrm{wg}}=$ $a x b$. In contrast, the radiation-pressure-induced SBS coupling is a strictly geometry-dependent effect, whose SBScoupling strength scales as $A_{\mathrm{wg}}^{-2}$. This scale dependence is seen from the fact that radiation pressure exerted by a guided mode scales as $A_{\mathrm{wg}}^{-1}$ [23]. It is apparent from the distinct scaling of both radiation-pressure and electrostrictively mediated SBS processes that it is no longer valid to treat SBS as a purely material-dependent quantity at subwavelength scales.

Intriguingly, despite the assumption of vanishing material nonlinearities in the treatment of the radiation-pressure component of the SBS gains, tremendous Brillouin nonlinearities are found. Hence, as a remarkable corollary, we find that strong nanoscale light-boundary interactions result in tremendous radiation-pressure-induced nonlinearities, even in the absence of any intrinsic material nonlinearity.

From fundamental considerations, one can see that Brillouin nonlinearities cannot be treated as intrinsic to a nonlinear medium because Brillouin processes involve coupling to collective elastic-wave excitations (or phonons) that are shaped by the extrinsic (or geometric) properties of the system. Furthermore, while the photoelastic- (or electrostrictive-) medium response is intrinsic to an electronic medium, radiation pressure gives rise to entirely new forms of nonlinearity produced solely by extrinsic geometric properties. It bears mentioning that recent reformulations of effective nonlinearities within nanoscale waveguides have accurately captured the intrinsic electronic nonlinearities at subwavelength scales [51]. Such treatments are insufficient, however, to capture the physics of Brillouin scattering where strong interactions with boundaries occur. Within high-confinement systems, the boundaries act to strongly shape the optical fields as well as the frequency and mode distribution of the resonantly excited phonons. Additionally, the strong interaction of light with boundaries yields new contributions to optical forces which dictate the strength and nature of photon-phonon coupling. Thus, explicit treatment of the underlying physics of photon-phonon coupling must always be made. Such treatment generally requires $a b$ initio formulation of Brillouin nonlinearities on a case-by-case basis.

\section{DISCUSSION}

The multiscale model of Sec. II builds on an impressive body of theoretical and experimental work which demonstrates accurate prediction of SBS within microscale systems, including the work of [1-21,25,42,43,52,53], and many others. Our multiscale treatment fundamentally differs from previous work in that it (1) captures Brillouin nonlinearities associated with nonlinear polarization currents at boundaries, and (2) accounts for new contributions to Brillouin gain resulting from radiation pressures. Through previous microscale treatments of SBS, radiation pressures and boundary-induced nonlinear couplings have been neglected.

For simplicity, we have assumed a frequencyindependent phononic-material loss, $Q$ of 1000 throughout this paper. However, since $G_{\mathrm{B}}$ increases linearly with phononic $\operatorname{loss} Q$, it is important to note that the $Q$ varies greatly with the type of elastic mode and can exceed the $Q=1000$ assumed here. For instance, the E2 wine-glass mode excited in the FSBS case is not limited by thermoelastic damping and results in a far higher quality factor $(Q \cong 3000)$ leading to an FSBS gain of $6.9 \times$ $10^{5} \mathrm{~m}^{-1} \mathrm{~W}^{-1}$ under ideal experimental conditions [49]. Moreover, since the phonon losses of silicon roll off rapidly at low temperatures [54], cryogenic cooling of such chip-scale waveguides could increase phononic $Q$ s by factors of 10-100, yielding an FSBS gain of between $10^{6}-10^{7} \mathrm{~m}^{-1} \mathrm{~W}^{-1}$. Thus, depending on the temperature of operation, these radically enhanced SBS processes can be between $10^{5}-10^{7}$ times stronger than those within typical silica-optical fibers. As a result, a nanoscale silicon waveguide of 100- $\mu \mathrm{m}$ length would yield an SBS nonlinearity equivalent to 10-1000 meters of conventional silica fiber.

Through examination of the silicon waveguide system examined here, the forces from both radiation pressure and electrostriction act to deform the waveguide in a similar manner, increasing the overall strength of photon-phonon coupling. However, these effects are not always additive. For instance, if the waveguide dimension or the mode shape is different from that examined here, radiation pressure can reduce the overall SBS coupling in many instances. If another core material such as Ge is considered, the lateral forces of radiation pressure and electrostriction will generally take on an opposite sign, producing destructive 
interference between the two effects. Thus, careful consideration of the nontrivial interplay between radiation pressure and electrostriction is necessary when treating photon-phonon coupling at subwavelength scales.

In conclusion, we have shown that subwavelength field confinement produces a new regime of stimulated Brillouin scattering, where radiation pressure plays a critical role in mediating photon-phonon interactions. At these scales, strong interaction of light with the boundaries of a nanoscale waveguide radically enhances SBS through both electrostrictive- and radiation-pressure-induced optical forces. The increased effects of boundaries yield new geometric degrees of freedom through which photonphonon-coupling strength and frequency can be tailored. At nanoscales, backward SBS gains were shown to be $10^{4}$ times greater than that in conventional silica fibers, with 100 times larger values than predicted by conventional scalar SBS theories. Furthermore, radically enhanced forward SBS couplings were shown to overtake backward SBS coupling, producing a forward SBS gain of $10^{5}$ times larger than in any other known waveguide system. At low temperatures, the freezing out of thermal phonons reduces phonon-scattering losses, yielding an additional 10-100 times enhancement of the SBS gain.

Finally, we have developed a generalized full-vectorial multiscale treatment of SBS coupling which is applicable at any length scale. This treatment fundamentally differs from previous work in that it (1) captures Brillouin nonlinearities associated with nonlinear polarization currents at boundaries, and (2) accounts for new contributions to Brillouin gain resulting from radiation pressures. We have shown that the treatment of these effects is essential to studies of SBS at subwavelength scales. This full-vectorial treatment of SBS interactions is applicable to periodic and translationally invariant waveguide systems and provides an exact means of treating both intermode and intramode coupling through SBS processes. The enhanced SBS couplings identified in this new regime of boundarymediated interactions could enable efficient stimulated Brillouin scattering for the first time in silicon waveguides, leading to practical chip-scale realization of slow light $[5,6]$, dynamically tailorable effective susceptibilities [5,6], and nonreciprocal nonlinear processes on optical chips [40].

\section{ACKNOWLEDGMENTS}

Sandia Laboratory is operated by Sandia Co., a Lockheed Martin Company, for the U.S. Department of Energy's NNSA under Contract No. DE-AC0494AL85000. This work was supported by the DDRE under Air Force Contract No. FA8721-05-C-000, the MesoDynamic Architectures program at DARPA under the direction of Dr. J. L. Rogers, and Sandia's Laboratory Directed Research and Development program under Dr. W. Hermina. We thank H. Shin, R. Olsson, I. El-Kady, M.
Eichenfield, R. W. Boyd, and E. P. Ippen for helpful technical discussions.

\section{APPENDIX A: COMPARISON WITH CONVENTIONAL SBS TREATMENTS}

Within microscale waveguides and optical fibers, the time-harmonically modulated force densities, which dictated the strength of photon-phonon coupling, are produced almost exclusively by electrostrictive interactions within the volume of the waveguide [2]. In these systems, photonphonon coupling is typically dominated by longitudinal guided elastic waves, reminiscent of bulk acoustic waves. Thus, the photoelastic properties of the waveguide material determine the strength of the SBS gain, yielding an SBS gain coefficient, $G_{\mathrm{B}}$ of the form [2]

$$
G_{\mathrm{B}}=\frac{\omega_{\mathrm{s}}^{2}}{c^{2}} \frac{n^{7} p_{12}^{2}}{c \rho V_{L}}\left(\frac{Q}{\Omega}\right) \eta .
$$

Here, $n$ and $p_{12}$ are the material refractive index and photoelastic constant about frequency $\omega_{\mathrm{s}}, c$ is the speed of light in vacuum, $V_{\mathrm{L}}$ and $Q$ are the longitudinal sound velocity and phononic material quality-factor at phonon frequency $\Omega$, and $\rho$ is the material mass-density. In Eq. (A1), $\eta$ is a spatial overlap integral which converges to $\eta=A_{\mathrm{wg}}^{-1}$ in the plane-wave limit, where $A_{\mathrm{wg}}$ is the cross-sectional area of the waveguide. Though simple, this scalar formulation of SBS gain is very accurate within microscale systems [1,2]. It is important to note that the above relation predicts an SBS gain which is relatively insensitive to geometry, and determined only by the intrinsic properties of the waveguide material.

The trend of conventional SBS gain, shown as the gray curve of Figs. 5(a) and 5(b), was evaluated using Eq. (A1) with $\eta=(0.588 a b)^{-1}$ for all waveguide dimensions. This simplified form of the SBS gain shows perfect agreement with the more rigorous overlap integral formulation of $\eta$ for waveguide dimensions of several microns and larger, and it allows us to establish the trend of microscale SBS gain based on material properties. Following the analysis of Refs. [52,53], $\eta$ can be more rigorously formulated as

$$
\eta=\left[\int_{\mathrm{wg}} a(x, y) b(x, y) d A\right]^{2} .
$$

Here, $a(x, y)$ is the normalized form of the longitudinal elastic-wave displacement amplitude such that $\int_{\mathrm{wg}} a(x, y)$. $d A \equiv 1$, and $b(x, y)$ is the power-normalized intensity distribution of the guided optical mode. Note, this integral form of $\eta^{-1}$ has units of area, and approaches $\eta=A_{\mathrm{wg}}^{-1}$ in the plane-wave limit. When Eq. (A1) is evaluated with the form of $\eta$ defined earlier, this formulation of SBS can be seen to be equivalent to that of Refs. [52,53], which is known to be remarkably accurate for the prediction of SBS gain within a wide range of microscale systems [2-6,11]. 


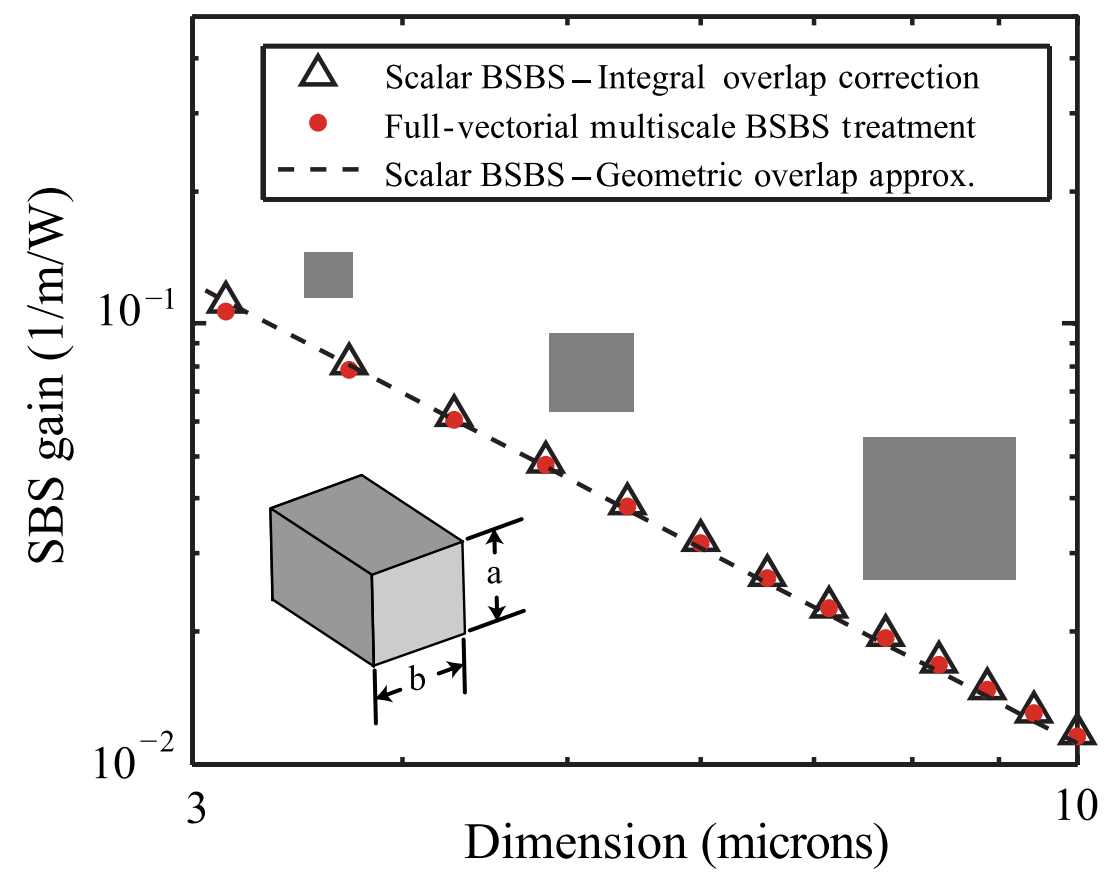

FIG. 6. Computed BSBS gain versus the waveguide dimension, $a$, for $b=0.93 a$. Comparison of a model based on MS-SBS theory (shown in red circles) is made with the integral form of the SBS gain (shown in triangles) and the simplified SBS trend (shown in dashes).

Note that the analytical formulation of multiscale SBS (MS-SBS), given in Eqs. (4) and (7) can be seen to produce the exact same form of the SBS gain described by Eqs. (A1) and (A2), demonstrating equivalence between MS-SBS and conventional models in the large-scale limit. Nevertheless, to validate the numerical models shown here, it is instructive to make a detailed comparison of simulations (based on the MS-SBS outlined in Secs. II, III, IV, V, and VI) with the proven microscale SBS theory outlined in this Appendix. Figure 6 shows one such comparison for the rectangular waveguide of the type described in Secs. III and IV. The results of MS-SBS theory (red circles), the integral form of the SBS gain described previously (triangles), and the simplified SBS trend (dashes) are all seen in Fig. 6. We see that a deviation of less than 1:1000 is seen between MS-SBS and the integral form of the SBS gain for dimensions between $4-10 \mu \mathrm{ms}$, demonstrating convergence between the numerical model and the semianalytical microscale SBS theory. It should be noted that the MS-SBS theory (red circles) includes the effects of both electrostriction and radiation pressure. Thus, we see that the effects of radiation pressure are negligible at microscales.

\section{APPENDIX B: GENERAL FORM OF THE TIME-VARYING OPTICAL FORCES}

Here, we outline the basic methods that we use to treat the time-varying forces produced by electrostriction and radiation pressure. We assume that pump and Stokes waves simultaneously propagate within the waveguide, with com- plex guided-mode-field distributions of the form

$$
\begin{aligned}
\tilde{\mathbf{E}}_{\mathbf{p}}(\mathbf{r}, t) & =\tilde{\mathbf{E}}_{\mathbf{p}}(x, y) \cdot e^{i\left(\mathbf{k}_{\mathbf{p}} \cdot \mathbf{z}-\omega_{p} t\right)} \\
& =\tilde{A}_{p} \cdot \tilde{\mathbf{e}}_{\mathbf{p}}(x, y) e^{i\left(\mathbf{k}_{\mathbf{p}} \cdot \mathbf{z}-\omega_{p} t\right)}, \\
\tilde{\mathbf{E}}_{\mathbf{s}}(\mathbf{r}, t) & =\tilde{\mathbf{E}}_{\mathbf{s}}(x, y) \cdot e^{i\left(\mathbf{k}_{\mathbf{s}} \cdot \mathbf{z}-\omega_{s} t\right)} \\
& =\tilde{A}_{s} \cdot \tilde{\mathbf{e}}_{\mathbf{s}}(x, y) \cdot e^{i\left(\mathbf{k}_{\mathrm{s}} \cdot \mathbf{z}-\omega_{s} t\right)} .
\end{aligned}
$$

Above, the time-harmonic field amplitude is computed from the complex field amplitude as $\mathbf{E}_{\mathbf{j}}(\mathbf{r}, t)=$ $\operatorname{Re}\left[\tilde{\mathbf{E}}_{\mathbf{j}}(\mathbf{r}, t)\right]$. Above, $\tilde{\mathbf{e}}_{\mathbf{s}}(\mathbf{x}, \mathbf{y})$ and $\tilde{\mathbf{e}}_{\mathbf{p}}(\mathbf{x}, \mathbf{y})$ are powernormalized mode-field profiles, defined such that $\tilde{A}_{\mathrm{s}}$ and $\tilde{A}_{\mathrm{p}}$ can be expressed as $\tilde{A}_{\mathrm{s}}=\sqrt{P_{\mathrm{s}}} \cdot e^{i \theta_{s}}$ and $\tilde{A}_{\mathrm{p}}=\sqrt{P_{\mathrm{p}}} e^{i \theta_{p}}$. Note that $\tilde{\mathbf{e}}_{\mathbf{s}}(\mathbf{x}, \mathbf{y})$ and $\tilde{\mathbf{e}}_{\mathbf{p}}(\mathbf{x}, \mathbf{y})$ can represent any two optical modes with similar or distinct spatial profiles, allowing us to quite generally treat cross-polarization SBS coupling [43] and other nontrivial situations involving intermode coupling.

To evaluate the SBS gain via Eq. (7), we seek the general form of the time-varying electrostrictive- and radiationpressure-induced force densities. Within the waveguide, a total field distribution of the form $\mathbf{E}_{\mathbf{t}}(\mathbf{r}, t)=\mathbf{E}_{\mathbf{s}}(\mathbf{r}, t)+$ $\mathbf{E}_{\mathbf{p}}(\mathbf{r}, t)$ results in wave interference to produce an energy density and optical forces that are time-harmonically modulated with frequency $\Omega=\omega_{\mathrm{p}}-\omega_{\mathrm{s}}$. It is this timevarying component of the force that is responsible for photon-phonon coupling. 
The field-induced electrostrictive-body stress distribution produced by guided optical modes within a dielectric waveguide can be rigorously formulated in terms of the photoelastic-tensor components $p_{i j k l}$ and the medium dielectric tensor, $\epsilon_{i j}$, as [24]

$$
\left\langle\sigma_{k l}^{\mathrm{es}}\right\rangle=-\frac{1}{2} \epsilon_{o} \sum_{k l}\left[\epsilon_{i j} p_{i j k l} \epsilon_{k l}\right]\left\langle E_{k}(t) E_{l}(t)\right\rangle .
$$

Here, $\langle\ldots\rangle$ denotes a time averaging. For the treatment of SBS, we examine the time-varying component of the optical forces at the beat frequency, $\Omega$.

While the approach for deriving the optical force is identical within media of any symmetry, we limit our attention to electrostrictive forces produced within cubic media. Within cubic media, one can show that the timeaveraged electrostrictive-body stress density is of the form [24]

$$
\left\langle\sigma_{k l}^{\mathrm{es}}\right\rangle=-\frac{1}{4} \epsilon_{o} n_{1}^{4} \sum_{i j} p_{i j k l} \operatorname{Re}\left[\tilde{E}_{i} \tilde{E}_{j}^{*}\right]
$$

Above, averaging at the time scale of the optical carrier frequency is obtained by replacing $\left\langle E_{k}(t) E_{l}(t)\right\rangle$ with $\operatorname{Re}\left[\tilde{E}_{i} \tilde{E}_{j}^{*}\right] / 2$. The electrostrictively induced force density is computed from the divergence of $\sigma_{k l}^{\mathrm{es}}$ as $f_{l}^{\mathrm{es}}=-\partial_{k} \sigma_{k l}^{\mathrm{es}}$. Thus, the force density of electrostriction is

$$
\left\langle f_{l}^{\mathrm{es}}\right\rangle=\left\langle-\partial_{k} \sigma_{k l}^{\mathrm{es}}\right\rangle=\frac{\epsilon_{o} n_{1}^{4}}{4} \sum_{k} \sum_{i j} p_{i j k l} \operatorname{Re}\left[\partial_{k} \tilde{E}_{i} \tilde{E}_{j}^{*}\right] .
$$

Substituting the total complex field amplitude using Eqs. (B1) and (B2) into Eq. (B5) and neglecting terms which do not vary harmonically with frequency $\Omega$, we find a temporally modulated force density of the form

$$
\begin{aligned}
& \mathbf{f}_{\boldsymbol{\Omega}}^{\mathbf{e s}}(\mathbf{r}, t)=\operatorname{Re}\left[\tilde{\mathbf{f}}_{\boldsymbol{\Omega}}^{\text {es }}(\mathbf{x}, \mathbf{y}) e^{i(\Delta \mathbf{k} \cdot \mathbf{z}-\boldsymbol{\Omega} t)}\right] \\
& =\operatorname{Re}\left[\tilde{\mathbf{f}}_{\mathbf{n}}^{\text {es }}(\mathbf{x}, \mathbf{y}) 2 \sqrt{P_{\mathrm{s}} P_{\mathrm{p}}} e^{i(\Delta \mathbf{k} \cdot \mathbf{z}-\Omega t)}\right] .
\end{aligned}
$$

Here, $\tilde{\mathbf{f}}_{\boldsymbol{\Omega}}^{\text {es }}(\mathbf{x}, \mathbf{y})=\tilde{\mathbf{f}}_{\mathbf{n}}^{\text {es }}(\mathbf{x}, \mathbf{y}) 2 \sqrt{P_{\mathrm{s}} P_{\mathrm{p}}}$, where $\tilde{\mathbf{f}}_{\mathbf{n}}^{\text {es }}$ is the power-normalized time-harmonic component of the electrostrictive force density, and $\Delta \mathbf{k}=\left(\mathbf{k}_{\mathbf{p}}-\mathbf{k}_{\mathbf{s}}\right)$. The timeharmonic force density, derived in this way, is a completely general form of the force density for application to both cubic and isotropic dielectric systems. Note that since the time-varying force density exhibits a longitudinal spatial modulation associated with $\Delta \mathbf{k}$, phase-matching requires that the excited phonon wave vector is given by $\mathbf{K}=\boldsymbol{\Delta} \mathbf{k}$.

The time-averaged force density from radiation pressure, $\left\langle f_{j}^{\mathrm{rp}}\right\rangle$, can be treated following a similar procedure. The force density associated with radiation pressure can be computed from the divergence of the Maxwell stress tensor as

$$
\left\langle f_{j}^{\mathrm{rp}}\right\rangle=\left\langle\partial_{i} T_{i j}\right\rangle=\frac{1}{2} \cdot \operatorname{Re}\left[\partial_{i} \tilde{T}_{i j}\right]
$$

where $\tilde{T}_{i j}$ is the Maxwell stress tensor of the form, $\tilde{T}_{i j}=$ $\epsilon_{o} \epsilon\left[\tilde{E}_{i} \tilde{E}_{j}^{*}-\frac{1}{2} \delta_{i j}|\tilde{E}|^{2}\right]+\mu_{o} \mu\left[\tilde{H}_{i} \tilde{H}_{j}^{*}-\frac{1}{2} \delta_{i j}|\tilde{H}|^{2}\right]$. Again, substituting the complex field amplitudes of Eqs. (B1) and (B2) into Eq. (B8), and neglecting terms which do not vary harmonically with frequency $\Omega$, we have a temporally modulated force density of the form

$$
\begin{aligned}
& \mathbf{f}_{\boldsymbol{\Omega}}^{\mathbf{r p}}(\mathbf{r}, t)=\operatorname{Re}\left[\tilde{\mathbf{f}}_{\boldsymbol{\Omega}}^{\mathbf{r p}}(\mathbf{x}, \mathbf{y}) e^{i(\boldsymbol{\Delta} \mathbf{k} \cdot \mathbf{z}-\Omega t)}\right] \\
= & \operatorname{Re}\left[\tilde{\mathbf{f}}_{\mathbf{n}}^{\mathbf{r p}}(\mathbf{x}, \mathbf{y}) 2 \sqrt{P_{s} P_{p}} e^{i(\Delta \mathbf{k} \cdot \mathbf{z}-\Omega t)}\right] .
\end{aligned}
$$

Here, $\tilde{\mathbf{f}}_{\mathbf{\Omega}}^{\mathrm{rp}}(\mathbf{x}, \mathbf{y})=\tilde{\mathbf{f}}_{\mathbf{n}}^{\mathrm{rp}}(\mathbf{x}, \mathbf{y}) 2 \sqrt{P_{\mathrm{s}} P_{\mathrm{p}}}$, where $\tilde{\mathbf{f}}_{\mathbf{n}}^{\mathrm{rp}}$ is the powernormalized time-harmonic component of the radiationpressure-induced force density. The time-harmonic force density, derived in this way, is valid for treatment of forces in dielectric systems where free charges are absent.

Thus, the total time-varying force, $\mathbf{f}_{\boldsymbol{\Omega}}(\mathbf{r}, t)$, is produced by a coherent superposition of electrostrictive forces and radiation pressure, and is of the form

$$
\mathbf{f}_{\mathbf{\Omega}}(\mathbf{r}, t)=\operatorname{Re}\left[\tilde{\mathbf{f}}_{\mathbf{n}}(\mathbf{x}, \mathbf{y}) 2 \sqrt{P_{s} P_{p}} e^{i(\Delta \mathbf{k} \cdot \mathbf{z}-\Omega t)}\right],
$$

where $\tilde{\mathbf{f}}_{\mathbf{n}}(\mathbf{x}, \mathbf{y})=\tilde{\mathbf{f}}_{\mathbf{n}}^{\text {rp }}(\mathbf{x}, \mathbf{y})+\tilde{\mathbf{f}}_{\mathbf{n}}^{\text {es }}(\mathbf{x}, \mathbf{y})$.

[1] R. Boyd, Nonlinear Optics (Academic Press, Boston, 2009), 3rd ed., Chap. 9.

[2] G. Agrawal, Nonlinear Fiber Optics (Academic Press, San Diego, 1995).

[3] R. Chiao, C. Townes, and B. Stoicheff, Stimulated Brillouin Scattering and Coherent Generation of Intense Hypersonic Waves, Phys. Rev. Lett. 12, 592 (1964).

[4] E. Ippen and R. Stolen, Stimulated Brillouin Scattering in Optical Fibers, Appl. Phys. Lett. 21, 539 (1972).

[5] Y. Okawachi, M. Bigelow, J. Sharping, Z. Zhu, A. Schweinsberg, D. Gauthier, R. Boyd, and A. Gaeta, Tunable All-Optical Delays via Brillouin Slow Light in an Optical Fiber, Phys. Rev. Lett. 94, 153902 (2005).

[6] K. Song, K. Abedin, K. Hotate, M. González Herráez, and L. Thévenaz, Highly Efficient Brillouin Slow and Fast Light Using $\mathrm{As}_{2} \mathrm{Se}_{3}$ Chalcogenide Fiber, Opt. Express 14, 5860 (2006).

[7] R. Pant, M. Stenner, M. Neifeld, and D. Gauthier, Optimal Pump Profile Designs for Broadband SBS Slow-Light Systems, Opt. Express 16, 2764 (2008).

[8] K. Song, M. Herráez, and L. Thévenaz, Observation of Pulse Delaying and Advancement in Optical Fibers Using Stimulated Brillouin Scattering, Opt. Express 13, 82 (2005).

[9] K. Nelson, Stimulated Brillouin Scattering and Optical Excitation of Coherent Shear Waves, J. Appl. Phys. 53, 6060 (1982).

[10] P. Dainese, P. Russell, N. Joly, J. Knight, G. Wiederhecker, H. Fragnito, V. Laude, and A. Khelif, Stimulated Brillouin Scattering from Multi-GHz-Guided Acoustic Phonons in Nanostructured Photonic Crystal Fibres, Nature Phys. 2, 388 (2006). 
[11] R. Shelby, M. Levenson, and P. Bayer, Guided AcousticWave Brillouin Scattering, Phys. Rev. B 31, 5244 (1985).

[12] A. Kobyakov, M. Sauer, and D. Chowdhury, Stimulated Brillouin Scattering in Optical Fibers, , Adv. Opt. Photon. 2, 1 (2009).

[13] M. S. Kang, A. Nazarkin, A. Brenn, and P. S. J. Russell, Tightly Trapped Acoustic Phonons in Photonic Crystal Fibres as Highly Nonlinear Artificial Raman Oscillators, Nature Phys. 5, 276 (2009).

[14] N. Olsson and J. Van Der Ziel, Fibre Brillouin Amplifier with Electronically Controlled Bandwidth, Electron. Lett. 22, 488 (1986).

[15] R. Pant, C. Poulton, D. Choi, H. Mcfarlane, S. Hile, E. Li, L. Thévenaz, B. Luther-Davies, S. Madden, and B. Eggleton, On-Chip Stimulated Brillouin Scattering, Opt. Express 19, 8285 (2011).

[16] D. Hon, Pulse Compression by Stimulated Brillouin Scattering, Opt. Lett. 5, 516 (1980).

[17] T. Tanemura, Y. Takushima, and K. Kikuchi, Narrowband Optical Filter, with a Variable Transmission Spectrum, Using Stimulated Brillouin Scattering in Optical Fiber, Opt. Lett. 27, 1552 (2002).

[18] Z. Zhu, D. Gauthier, and R. Boyd, Stored Light in an Optical Fiber via Stimulated Brillouin Scattering, Science 318, 1748 (2007).

[19] M. Tomes and T. Carmon, Photonic MicroElectromechanical Systems Vibrating at X-Band (11-GHz) Rates, Phys. Rev. Lett. 102, 113601 (2009).

[20] J. Wang, Y. Zhu, R. Zhang, and D. Gauthier, FSBS Resonances Observed in a Standard Highly Nonlinear Fiber, Opt. Express 19, 5339 (2011).

[21] P. Rakich, Y. Fink, and M. Soljačić, Efficient Mid-IR Spectral Generation via Fifth-Order Cascaded-Raman Amplification in Silica Fibers, Opt. Lett. 33, 1690 (2008).

[22] M. Povinelli, M. Loncar, M. Ibanescu, E. Smythe, S. Johnson, F. Capasso, and J. Joannopoulos, EvanescentWave Bonding between Optical Waveguides, Opt. Lett. 30, 3042 (2005).

[23] P. Rakich, Z. Wang, and P. Davids, Scaling of Optical Forces in Dielectric Waveguides: Rigorous Connection between Radiation Pressure and Dispersion, Opt. Lett. 36, 217 (2011).

[24] P. Rakich, P. Davids, and Z. Wang, Tailoring Optical Forces in Waveguides through Radiation Pressure and Electrostrictive Forces, Opt. Express 18, 14439 (2010).

[25] T. Kippenberg and K. Vahala, Cavity Optomechanics: Back-Action at the Mesoscale, Science 321, 1172 (2008).

[26] M. Eichenfield, C. Michael, R. Perahia, and O. Painter, Actuation of Micro-Optomechanical Systems via CavityEnhanced Optical Dipole Forces, Nature Photon. 1, 416 (2007).

[27] P. Rakich, M. Popović, M. Soljačić, and E. Ippen, Trapping, Corralling and Spectral Bonding of Optical Resonances through Optically Induced Potentials, Nature Photon. 1, 658 (2007).

[28] M. Li, W. H. P. Pernice, and H.X. Tang, Tunable Bipolar Optical Interactions between Guided Lightwaves, Nature Photon. 3, 464 (2009).

[29] W. H. P. Pernice, M. Li, and H. X. Tang, Optomechanical Coupling in Photonic Crystal Supported Nanomechanical Waveguides, Opt. Express 17, 12424 (2009).
[30] J. Roels, I. De Vlaminck, L. Lagae, B. Maes, D. Van Thourhout, and R. Baets, Tunable Optical Forces between Nanophotonic Waveguides, Nature Nanotech. 4, 510 (2009).

[31] P. Rakich, M. Popović, and Z. Wang, General Treatment of Optical Forces and Potentials in Mechanically Variable Photonic Systems, Opt. Express 17, 18116 (2009).

[32] M. Eichenfield, R. Camacho, J. Chan, K. J. Vahala, and O. Painter, A Picogram- and Nanometre-Scale PhotonicCrystal Optomechanical Cavity, Nature (London) 459, 550 (2009).

[33] G. Wiederhecker, L. Chen, A. Gondarenko, and M. Lipson, Controlling Photonic Structures Using Optical Forces, Nature (London) 462, 633 (2009).

[34] P. Deotare, M. McCutcheon, I. Frank, M. Khan, and M. Lončar, Coupled Photonic Crystal Nanobeam Cavities, Appl. Phys. Lett. 95, 031102 (2009).

[35] Q. Lin, J. Rosenberg, X. Jiang, K. Vahala, and O. Painter, Mechanical Oscillation and Cooling Actuated by the Optical Gradient Force, Phys. Rev. Lett. 103, 103601 (2009).

[36] I. Grudinin, H. Lee, O. Painter, and K. Vahala, Phonon Laser Action in a Tunable Two-Level System, Phys. Rev. Lett. 104, 83901 (2010).

[37] A. Safavi-Naeini, T. Alegre, J. Chan, M. Eichenfield, M. Winger, Q. Lin, J. Hill, D. Chang, and O. Painter, Electromagnetically Induced Transparency and Slow Light with Optomechanics, Nature (London) 472, 69 (2011).

[38] Q. Lin, J. Rosenberg, D. Chang, R. Camacho, M. Eichenfield, K. Vahala, and O. Painter, Coherent Mixing of Mechanical Excitations in Nano-optomechanical Structures, Nature Photon. 4, 236 (2010).

[39] M. Li, W.H.P. Pernice, and H.X. Tang, Broadband AllPhotonic Transduction of Nanocantilevers, Nature Nanotech. 4, 377 (2009).

[40] M. S. Kang, A. Butsch, and P. S. J. Russell, Reconfigurable Light-Driven Opto-Acoustic Isolators in Photonic Crystal Fibre, Nature Photon. 5, 549 (2011).

[41] S. G. Johnson, M. Ibanescu, M. A. Skorobogatiy, O. Weisberg, J. D. Joannopoulos, and Y. Fink, Perturbation Theory for Maxwell's Equations with Shifting Material Boundaries, Phys. Rev. E 65, 066611 (2002).

[42] Y.R. Shen and N. Bloembergen, Theory of Stimulated Brillouin and Raman Scattering, Phys. Rev. 137, A1787 (1965).

[43] M. Kang, A. Brenn, and P. St. J. Russell, All-Optical Control of Gigahertz Acoustic Resonances by Forward Stimulated Interpolarization Scattering in a Photonic Crystal Fiber, Phys. Rev. Lett. 105, 153901 (2010).

[44] L.D. Landau and E. M. Lifshitz, Elasticity Theory (Pergamon Press, Oxford, United Kingdom, 1975).

[45] L. Hounsome, R. Jones, M. Shaw, and P. Briddon, Photoelastic Constants in Diamond and Silicon, Phys. Status Solidi A 203, 3088 (2006).

[46] E. Dieulesaint and D. Royer, Elastic Waves in Solids II: Generation, Acousto-Optic Interaction, Applications (Springer, New York, 2000).

[47] P. Loh, A. Oskooi, M. Ibanescu, M. Skorobogatiy, and S. 
Johnson, Fundamental Relation between Phase and Group Velocity, and Application to the Failure of Perfectly Matched Layers in Backward-Wave Structures, Phys. Rev. E 79, 065601 (2009).

[48] J. Gordon, Radiation Forces and Momenta in Dielectric Media, Phys. Rev. A 8, 14 (1973).

[49] S. A. Chandorkar, R. N. Candler, A. Duwel, R. Melamud, M. Agarwal, K. E. Goodson, and T. W. Kenny, Multimode Thermoelastic Dissipation, J. Appl. Phys. 105, 043505 (2009).

[50] COMSOL AB, COMSOL 3.5: Structural Mechanics Module User's Guide, (COMSOL AB, 2008).

[51] S. Afshar V and T. Monro, A Full Vectorial Model for
Pulse Propagation in Emerging Waveguides with Subwavelength Structures Part I: Kerr Nonlinearity, Opt. Express 17, 2298 (2009).

[52] J. Botineau, E. Picholle, and D. Bahloul, Effective Stimulated Brillouin Gain in Singlemode Optical Fibres, Electron. Lett. 31, 2032 (1995).

[53] A. McCurdy, Modeling of Stimulated Brillouin Scattering in Optical Fibers with Arbitrary Radial Index Profile, J. Lightwave Technol. 23, 3509 (2005).

[54] H.-Y. Hao and H. Maris, Dispersion of the LongWavelength Phonons in Ge, Si, GaAs, Quartz, and Sapphire, Phys. Rev. B 63, 224301 (2001). 